\title{
MODIFICACIÓN DE LAS PROPIEDADES ESTRUCTURALES, ELÉCTRICAS Y MAGNÉTICAS DEL BiFeO 3 POR LA INCORPORACIÓN DE Ba Y Nb
}

\author{
Cristian Andres Narváez, Claudia Fernanda Villaquirán Raigozaa,* y Aida Patricia González Nieva ${ }^{\mathrm{b}}$ \\ aDepartamento de Física, Universidad del Cauca. Popayán, Colombia

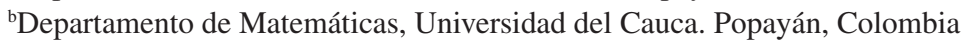

Recebido em 05/08/2016; aceito em 04/10/2016; publicado na web em 02/11/2016

\begin{abstract}
MODIFICATION OF STRUCTURAL, ELECTRICAL AND MAGNETIC PROPERTIES OF $\mathrm{BiFeO}_{3}$ BY INCORPORATING Ba AND Nb. Bismuth ferrite (BFO) and Ba-Nb-substituted $\mathrm{BFO}$ with compositions $\mathrm{Bi}_{1-\mathrm{x}} \mathrm{Ba}_{\mathrm{x}} \mathrm{FeO}_{3}\left(\mathrm{x}=0,0.10,0.15\right.$ y 0.20 ), $\mathrm{BiFe}_{1-\mathrm{y}} \mathrm{Nb}_{\mathrm{y}} \mathrm{O}_{3}$ $(\mathrm{y}=0,0.03,0.06$ y 0.09$)$ y $\mathrm{Bi}_{1-\mathrm{x}} \mathrm{Ba}_{\mathrm{x}} \mathrm{Fe}_{1-\mathrm{y}} \mathrm{Nb}_{\mathrm{y}} \mathrm{O}_{3}(\mathrm{x}=0.10$ (y=0, 0.03, 0.06 y 0.09$)$ powders were synthesized using the citrate-gel method. Infrared spectroscopy indicated shifting of $\mathrm{Bi} / \mathrm{Ba}-\mathrm{O}$ and $\mathrm{Fe} / \mathrm{Nb}-\mathrm{O}$ bond vibrations and a structural transition was observed by X-ray diffraction. A Williamson-Hall (W-H) analysis revealed that $\mathrm{Ba} / \mathrm{Nb}$ dopant reduces the particle size and increases the strain crystalline lattice. Impedance analysis suggests that electrical relaxation process is temperature dependent, for all compositions obtained. Furthermore, electrical conductivity increases with temperature showing a typical negative temperature coefficient of the resistance (NTCR) analogous to a semiconductor. Magnetization v magnetic field curves reveal weak ferromagnetic behaviour within the $\mathrm{BiFeO}_{3}$ doped with $\mathrm{Ba}$ and $\mathrm{Nb}$, and co-doped with $\mathrm{Ba}-\mathrm{Nb}$, measured at room temperature.
\end{abstract}

Keywords: multiferroic; citrate-gel; W-H analysis; magnetic properties.

\section{INTRODUCCIÓN}

Existen muy pocos materiales multiferroicos tanto en su forma natural como sintetizados en un laboratorio, un material representativo de este grupo es la ferrita de bismuto, $\mathrm{BiFeO}_{3}$ (BFO), que presenta ventajas sobre otros compuestos multiferroicos debido a sus elevadas temperaturas de Curie y de Néel $\left(\mathrm{T}_{\mathrm{C}} \sim 1100 \mathrm{~K}, \mathrm{~T}_{\mathrm{N}} \sim 640 \mathrm{~K}\right)$. El $\mathrm{BFO}$ ha despertado interés en las ciencias básicas y ha demostrado un gran potencial para nuevas aplicaciones, debido a que presenta propiedades ferromagnéticas (ordenamiento antiferromagnético tipo G) y ferroeléctricas a temperatura ambiente. ${ }^{1}$ El problema principal de este sistema es la dificultad de obtenerlo monofásico por métodos convencionales ya que al sintetizarlo se forman simultáneamente diversas fases secundarias como $\mathrm{Bi}_{2} \mathrm{O}_{3}, \mathrm{Bi}_{2} \mathrm{Fe}_{4} \mathrm{O}_{9}$ y $\mathrm{Bi}_{25} \mathrm{FeO}_{39}$; una de las opciones para eliminar la presencia de fases secundarias es su dopaje con cationes que sustituyan los sitios A y B en su estructura y a su vez, favorezca sus propiedades físicas. Un aumento de la magnetización fue reportado para cerámicas de $\mathrm{BiFeO}_{3}$ dopadas, indicando una forma posible de suprimir la modulación de espines en soluciones sólidas $\mathrm{Bi}_{1-\mathrm{x}} \mathrm{A}_{\mathrm{x}} \mathrm{FeO}_{3}(\mathrm{~A}=\mathrm{La}, \mathrm{Ba}, \mathrm{Pb}){ }^{2,3,4}$ Se encontró que el valor de la magnetización neta depende fuertemente de la clase de elemento diamagnético que se use como dopante; por ejemplo, la magnetización espontánea de las muestras dopadas con bario es al menos un orden de magnitud mayor que el observado para las soluciones sólidas resultado del dopaje con lantano con la misma concentración de dopante, ${ }^{2,4}$ la naturaleza de una correlación entre el tipo de dopante diamagnético y las propiedades magnéticas de las muestras aún no es muy clara. ${ }^{3}$ Recientemente, algunos investigadores estudiaron la sustitución de hierro por niobio para el BFO, reportando un gran aumento en la resistividad eléctrica de las cerámicas obtenidas, una débil polarización remanente y un comportamiento similar al ferromagnético. ${ }^{5}$ Otros investigadores prepararon cerámicas de BFO sustituyendo el hierro por niobio y cobalto, reportando pequeñas transiciones de fase estructurales, así como bajas pérdidas dieléctricas, ellos sugieren que el dopaje causa una distorsión de

*e-mail: gure@unicauca.edu.co los octaedros $\mathrm{FeO}_{6}$ y la modificación de la estructura de espines antiparalelos. ${ }^{6}$ En el desarrollo de esta investigación, se estudió el efecto del dopante sobre las propiedades estructurales, microestructurales, eléctricas y magnéticas en cerámicas y polvos cerámicos de los sistemas multiferroicos $\mathrm{Bi}_{1-\mathrm{x}} \mathrm{Ba}_{\mathrm{x}} \mathrm{FeO}_{3}(\mathrm{x}=0,0.10,0.15$ y 0.20), $\mathrm{BiFe}_{1-\mathrm{y}} \mathrm{Nb}_{\mathrm{y}} \mathrm{O}_{3}(\mathrm{y}=0,0.03,0.06$ y 0.09$)$ y $\mathrm{Bi}_{1-\mathrm{x}} \mathrm{Ba}_{\mathrm{x}} \mathrm{Fe}_{1-\mathrm{y}} \mathrm{Nb}_{\mathrm{y}} \mathrm{O}_{3}(\mathrm{x}=0,0.10$ $(\mathrm{y}=0,0.03,0.06$ y 0.09), sintetizados por el método gel-citrato. Los polvos cerámicos fueron caracterizados por espectroscopia infrarroja, difracción de rayos X, microscopía electrónica de barrido (MEB), la caracterización magnética fue realizada a través del análisis de curvas de histéresis obtenidas a través del magnetómetro tipo SQUID. Las muestras cerámicas fueron caracterizadas eléctricamente a través del análisis de las curvas de impedancia. El análisis del perfil de rayos $\mathrm{X}$ es una herramienta sencilla y potente para estimar el tamaño de cristalito y la deformación de la red. ${ }^{7}$ Entre los métodos disponibles para estimar estos dos valores están: la función pseudo-Voigt, el refinamiento Rietveld, y el análisis Warren Averbach. ${ }^{8,9,10} \mathrm{El}$ análisis de Williamson-Hall (W-H) es un método simple donde se realiza la deconvolución del ancho del perfil de difracción inducido tanto por el tamaño del cristalito como por la deformación, considerando la anchura de pico como una función de $2 \theta .{ }^{11}$ En el presente estudio, se utilizó el análisis W-H para estimar el tamaño de cristalito y la deformación de la red.

\section{PARTE EXPERIMENTAL}

Como fuente de cationes para la obtención de polvos cerámicos de BFO se emplearon los siguientes precursores: nitrato de bismuto (Merck-98\%), nitrato de hierro nonahidratado (Aldrich-99\%), nitrato de bario (Merck-99\%), ácido nítrico (Panreac-65\%), ácido cítrico (Merck-99.5\%), oxalato de Niobio (CBMM). El proceso gel citrato, utilizado en la síntesis de los polvos cerámicos, se realiza en un medio líquido conformado por ácido cítrico, en esta solución son solubles un amplio rango de sales metálicas, la presencia del ácido cítrico, facilita el proceso de poliesterificación; ${ }^{12,13}$ la mezcla, en agitación continua, lleva a la conformación de una disolución líquida transparente. Como en el sistema se encuentran presentes 
cationes, es posible que se conformen quelatos polibásicos y ácidos, que posteriormente, por calentamiento, experimenten polimerización, dando origen a una resina viscosa. ${ }^{14}$ La relación molar entre ácido cítrico y cationes, utilizada en el proceso de obtención de los polvos cerámicos fue 4:1. Inicialmente se disuelve el ácido cítrico en $10 \mathrm{~mL}$ de agua destilada sobre una plancha a $70{ }^{\circ} \mathrm{C}$ y en continúa agitación hasta que se obtiene una solución homogénea y transparente. Los precursores, previamente disueltos en una solución $3 \mathrm{~mol} \mathrm{~L}^{-1}$ de ácido nítrico, son adicionados a la disolución inicial obteniéndose una solución que debe ser transparente lo que confirma la no formación de precipitados. Luego la temperatura se aumentó a $120^{\circ} \mathrm{C}$ con el fin de evaporar el agua y facilitar el proceso de gelificación. Después de la obtención del gel, el sistema se trata a una temperatura $\sim 300^{\circ} \mathrm{C}$ en un horno hasta que experimenta un proceso de combustión que facilita la reacción y la obtención de polvos de fácil molienda. Los polvos obtenidos a través de este proceso contienen material orgánico por lo que son tratados térmicamente para lograr su eliminación y favorecer la cristalización del sistema; este tratamiento térmico se realizó en un horno a $750{ }^{\circ} \mathrm{C}$ durante tres horas. Los polvos cerámicos obtenidos se analizaron utilizando espectroscopia infrarroja con transformada de Fourier (FTIR) utilizando el espectrofotómetro Termo Electrón Nicolet IR200 FT-IR; mediante una interfaz que tiene un software denominado EZ-OMNIC, se obtuvo el espectro infrarrojo de la muestra; la deconvolución de las bandas presentes en los espectros fue realizada utilizando el software FITYK 0.8.6 (Marcin Wojdyr). La difracción de rayos-X fue realizada en un difractómetro de rayos X Philips, modelo PW3710, con radiación $\mathrm{CuK}_{\alpha}, \alpha_{1}=1.54056 \AA$ y $\alpha_{2}=1.54439 \AA$, con paso de $0.02^{\circ}$ y $2 \theta$ en un intervalo de $20^{\circ}$ a $60^{\circ}$. Las micrografías fueron obtenidas con el microscopio electrónico de barrido FEI modelo Nova Nano SEM 230 operando a 300 kV. Las curvas de histéresis magnética fueron obtenidas a temperatura ambiente bajo un campo magnético en el intervalo 0-10 kOe utilizando un magnetómetro de SQUID Quantum Design modelo MPMS 5S. Posteriormente con los polvos cerámicos se conformaron pastillas y se realizó la caracterización eléctrica con un analizador de impedancia 4192A LF en un rango de $5 \mathrm{~Hz}$ a13 MHz.

\section{RESULTADOS Y DISCUSIÓN}

En las Figuras 1(a) y (b) se observan los espectros infrarrojos de los polvos cerámicos de los sistemas $\mathrm{Bi}_{1-\mathrm{X}} \mathrm{Ba}_{\mathrm{X}} \mathrm{Fe}_{1-\mathrm{y}} \mathrm{Nb}_{\mathrm{y}} \mathrm{O}_{3}$ para diferentes concentraciones de bario y niobio. En ellas, se puede observar que, a excepción de las bandas presentes a $\sim 3500 \mathrm{~cm}^{-1}$ y a $\sim 1630 \mathrm{~cm}^{-1}$ que corresponden a modos de vibración del agua, ${ }^{15}$ sólo se presentan bandas relevantes en el rango comprendido entre

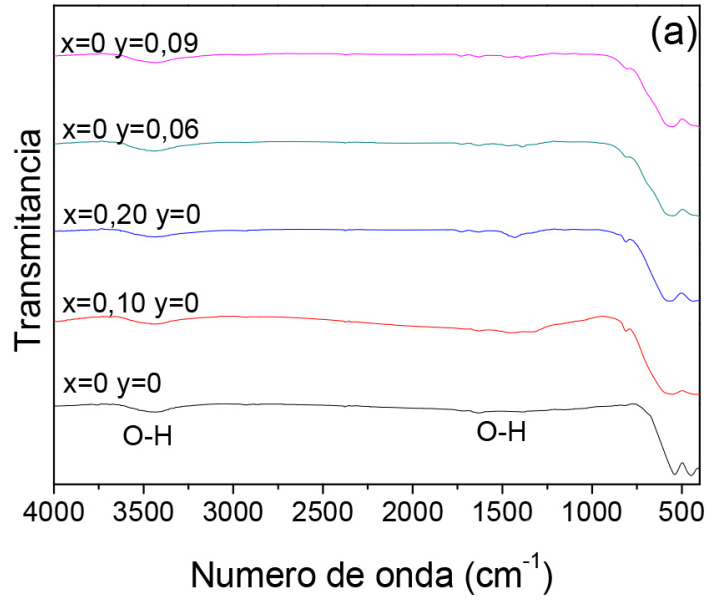

$1000 \mathrm{~cm}^{-1}-400 \mathrm{~cm}^{-1}$, correspondientes a los modos de vibración de los cationes de interés.

En los espectros de las Figuras 1(b) se observa adicionalmente a las bandas mencionadas, una banda a $\sim 1430 \mathrm{~cm}^{-1}$ correspondiente al enlace $\mathrm{C}=\mathrm{C}$ atribuido a los precursores utilizados en el proceso de síntesis, ${ }^{15}$ también se pueden identificar dos bandas características a $\sim 450 \mathrm{~cm}^{-1}$ y a $\sim 550 \mathrm{~cm}^{-1}$, que se pueden asociar a los modos "bending" del enlace O-Fe-O y "streching" del enlace $\mathrm{Fe}-\mathrm{O}$ respectivamente. ${ }^{16} \mathrm{~A}$ medida que se dopa con bario y niobio, estas bandas sufren un fuerte ensanchamiento; esta región será analizada con más detalle, mediante un proceso de deconvolución. La región comprendida entre $\sim 900$ $\mathrm{cm}^{-1}-400 \mathrm{~cm}^{-1}$ fue analizada mediante el proceso de deconvolución (Figura 2), el cual permite hacer un análisis detallado a cada uno de los espectros obtenidos para cada estequiometria. Se identificaron las bandas características con sus respectivos grupos funcionales. En el BFO sin dopaje (Figura 2a) se identificó una banda a $\sim 455 \mathrm{~cm}^{-1}$ 17 asociada a las vibraciones $\mathrm{O}-\mathrm{Fe}-\mathrm{O}$ "bending" del octaedro $\mathrm{FeO}_{6}$ y se puede relacionar con la existencia de la fase perovskita, la cual presenta este tipo de octaedros en el BFO, las bandas a $\sim 540, \sim 555$ y $\sim 579 \mathrm{~cm}^{-1}$, $^{18}$ están asociadas a las vibraciones Fe-O "stretching" características del octaedro $\mathrm{FeO}_{6}$ en los compuestos tipo perovskita y la banda a $\sim 810 \mathrm{~cm}^{-1}$ corresponde al modo vibracional stretching del enlace $\mathrm{Bi}-\mathrm{O},{ }^{19}$ con la incorporación de bario en la estructura, sistema $\mathrm{Bi}_{0.9} \mathrm{Ba}_{0.1} \mathrm{FeO}_{3}$ (Figura 2b), se evidencia la modificación en las bandas, este comportamiento puede ser explicado con base en la diferencia de los radios iónicos, $\mathrm{Bi}\left(\mathrm{r}_{\mathrm{Bi}+3}=1,40 \AA\right)^{20}$ y Ba $\left(\mathrm{r}_{\mathrm{Ba}+2}=1,61 \AA\right),{ }^{21}$ en coordinación 12 , lo que lleva naturalmente a una distorsión de la estructura debido a que el bario tiene un tamaño apreciablemente mayor que el bismuto, debido a esta distorsión, las bandas experimentan desplazamiento; la banda ubicada a $\sim 640 \mathrm{~cm}^{-1}$ está asociada a vibraciones Fe-O tipo "Stretching". ${ }^{18}$ Cuando se incorpora $6 \%$ de niobio al BFO (Figura 2c) aparece la banda a $\sim 650 \mathrm{~cm}^{-1}$ que se puede asociar al modo vibracional del octaedro $\mathrm{NbO}_{6},{ }^{22}$ la banda correspondiente al modo vibracional tipo "stretching" del Fe-O experimenta un corrimiento a $\sim 532 \mathrm{~cm}^{-1}$ lo que puede ser atribuido, en parte, a la diferencia en los radios iónicos del catión hospedero y del dopante, ${ }^{18}$ con coordinación $6, \mathrm{Fe}\left(\mathrm{r}_{\mathrm{Fe}+3}=0,55 \AA\right)$ y Nb $\left(\mathrm{r}_{\mathrm{Nb}+5}=0,64 \AA\right) .{ }^{23}$ Las Figuras 2(d), (e) y (f) muestran las deconvoluciones realizadas a los espectros del sistema con un contenido de bario fijo (10\%) y un contenido de niobio de 3\%, $6 \%$ y $9 \%$; en todas las Figuras se observa una banda ubicada a $\sim 447 \mathrm{~cm}^{-1}$ (O-Fe-O “Bending”), también se observa la presencia de una banda a $\sim 663 \mathrm{~cm}^{-1}$ en la Figura 2(d), no detectada en la Figuras 2(e) y (f), asociada también a los modos de vibración de los enlaces Fe-O. ${ }^{18} \mathrm{Al}$ dopar con el menor porcentaje de niobio $(\mathrm{y}=0,03)$, las bandas presentes en el material sin dopar

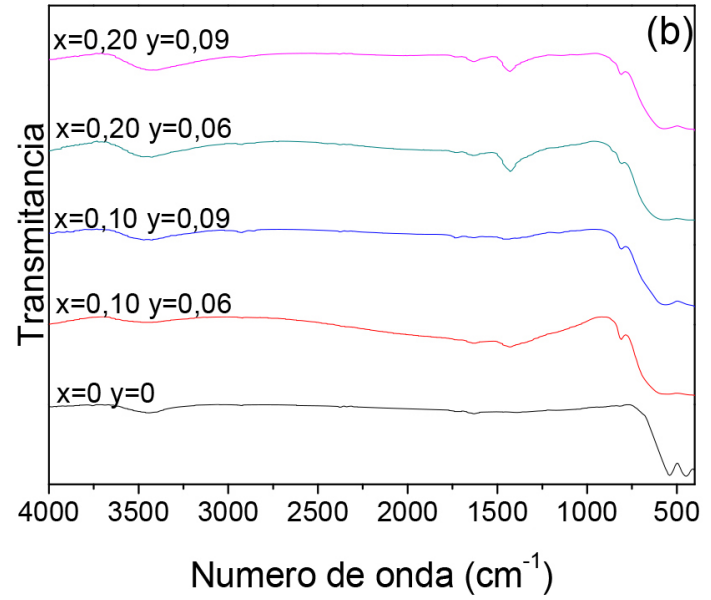

Figura 1. Espectros infrarrojos de los polvos cerámicos del sistema $\mathrm{Bi}_{1-x} \mathrm{Ba}_{x} \mathrm{Fe}_{1-y} \mathrm{Nb}_{y} \mathrm{O}_{3}$ para diferentes concentraciones de Bario y Niobio 
experimentan desplazamiento, la ubicada $\sim 810 \mathrm{~cm}^{-1}$ y asociada al modo vibracional del $\mathrm{Bi}-\mathrm{O}$ sufre un corrimiento a mayor número de onda $\left(\sim 814 \mathrm{~cm}^{-1}\right)$. Cuando $\mathrm{y}=0,06$ la banda ubicada a $\sim 663 \mathrm{~cm}^{-1}$ experimenta un desplazamiento a $\sim 671 \mathrm{~cm}^{-1}$ y aumenta ligeramente en anchura e intensidad.

Cuando $\mathrm{y}=0,09$ esta banda se desplaza hasta alcanzar un número de onda de $\sim 687 \mathrm{~cm}^{-1}$ y su intensidad aumenta, esta banda también está asociada con los modos vibracionales del catión dopante; para esta concentración, la banda ubicada a $\sim 579 \mathrm{~cm}^{-1}$ experimenta un desplazamiento a $\sim 586 \mathrm{~cm}^{-1}$ (mayor número de onda), esta banda está asociada al modo "stretching" de los enlaces Fe-O ${ }^{24} \mathrm{La}$ aparición de nuevas bandas a medida que se incrementa la concentración de BFO
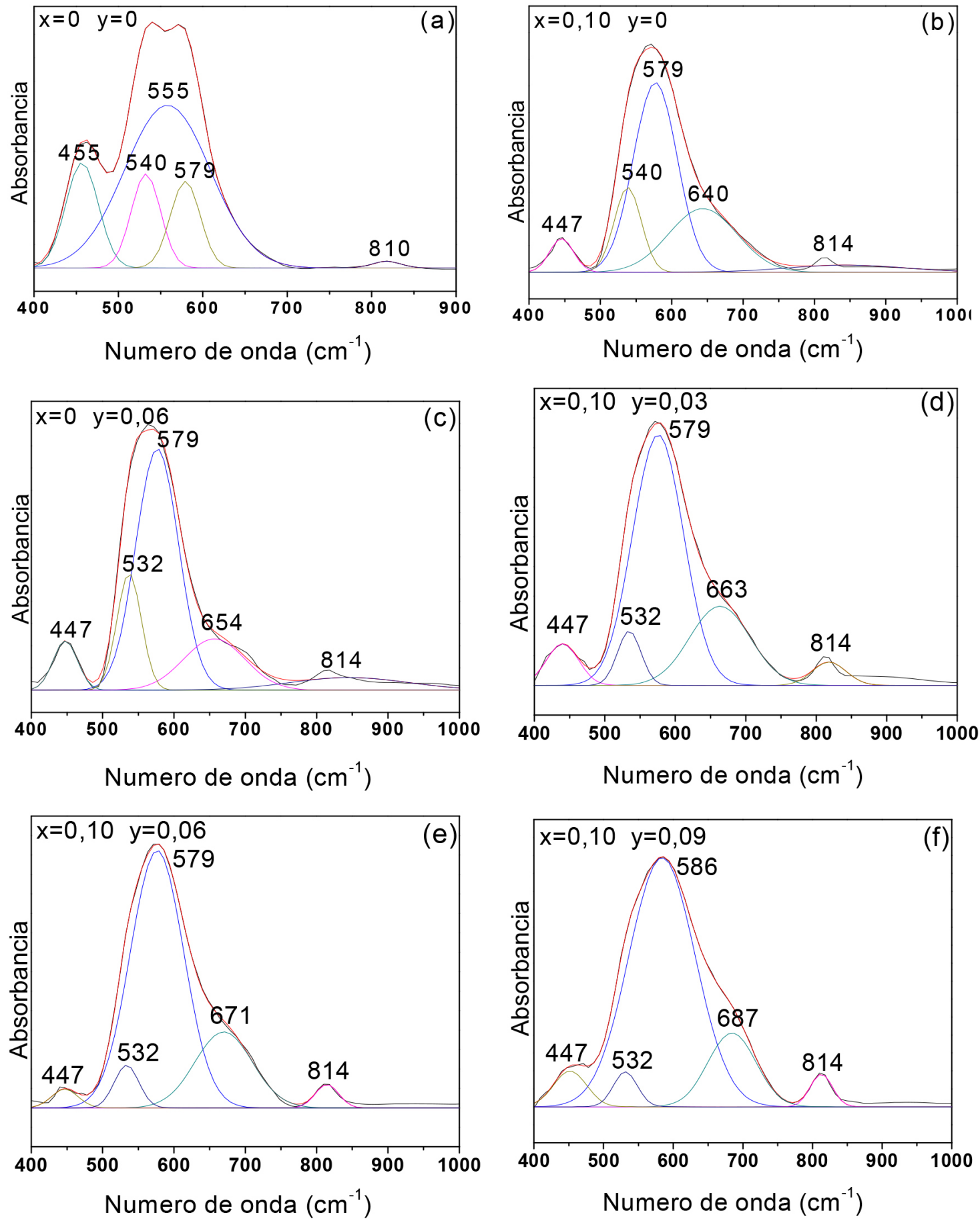

se da por el ordenamiento catiónico en posiciones equivalente y/o a la distorsión de la celda unitaria. ${ }^{25}$ El desplazamiento de las bandas, a medida que aumenta el porcentaje del dopante, puede ser debido a: (i) que la inclusión de iones de distinta naturaleza genera nuevos enlaces con una constante de fuerza diferente; las bandas del hierro sufren un corrimiento hacia números de ondas mayores, este comportamiento puede explicarse mediante la diferencia de electronegatividad, que es proporcional a la constante de fuerza y esta a su vez es un parámetro que mide la resistencia de una molécula a ser deformada. ${ }^{26}$ Como los enlaces $\mathrm{Fe}-\mathrm{O}$ tienen una diferencia de electronegatividad de 1,61 que es menor a la presentada por los enlaces $\mathrm{Nb}-\mathrm{O}(1,84)$, tendrán una mayor frecuencia de vibración; (ii) la incorporación de
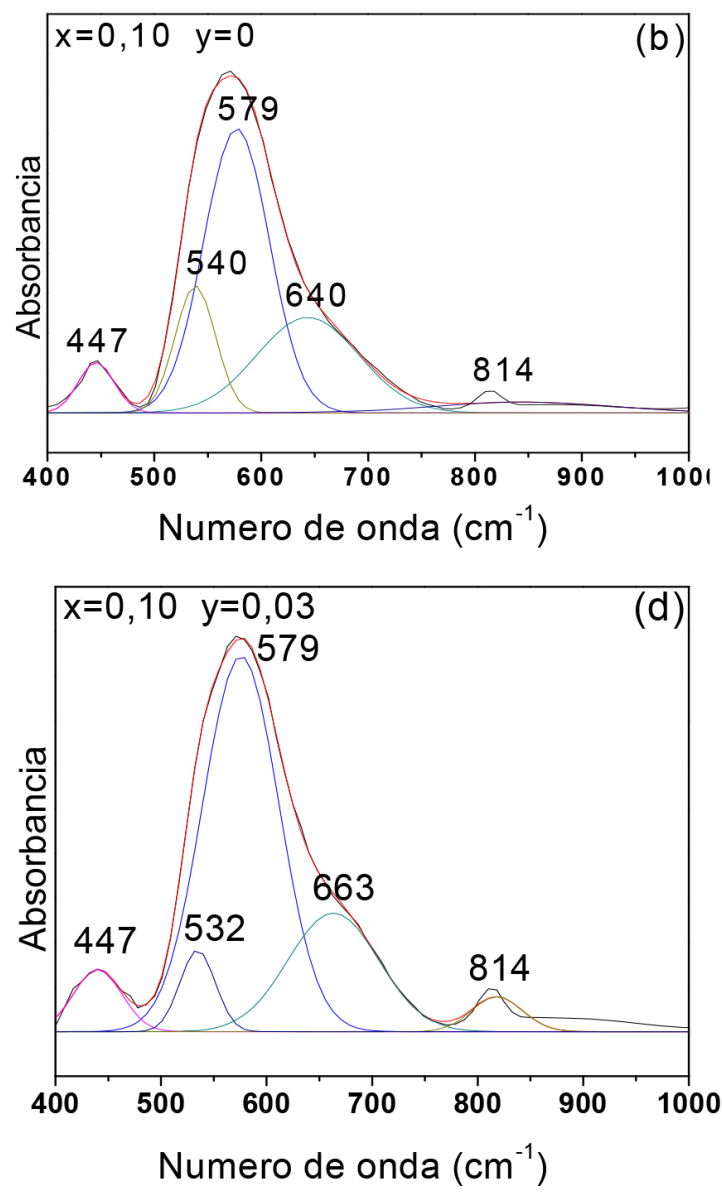

Figura 2. Deconvolución de los espectros FTIR del sistema $B i_{1-X} B a_{X} F e_{1-y} N b_{y} O_{3}$ para: (a) $x=0 y=0 ;$ (b) $x=0,10 y=0 ;(c) x=0 y=0,06$; (d) $x=0,10 y=0,03$; (e) $x=0,10 \quad y=0,06$ y (f) $x=0,10 \quad y=0,09$ 
$\mathrm{Ba}$ y $\mathrm{Nb}$ en la estructura del BFO hace que varíe la masa reducida del sistema; la diferencia de las masas atómicas que existe entre el Bi $\left(208,98 \mathrm{~g} \mathrm{~mol}^{-1}\right), \mathrm{Ba}\left(137,34 \mathrm{~g} \mathrm{~mol}^{-1}\right), \mathrm{Nb}\left(92,91 \mathrm{~g} \mathrm{~mol}^{-1}\right)$ y Fe $\left(55,84 \mathrm{~g} \mathrm{~mol}^{-1}\right)$ hacen que haya un desplazamiento de las bandas, ya que la frecuencia está relacionada con la masa reducida del sistema mediante la relación $v=\sqrt{\frac{k}{\mu}}$, donde $k$ es la constante de fuerza de los enlaces presentes y $\mu$ la masa reducida. ${ }^{27} \mathrm{La}$ aparición de nuevas bandas a medida que se incrementa la concentración de BFO se da por el ordenamiento catiónico en posiciones equivalentes y/o a la distorsión de la celda unitaria, ${ }^{25}$ lo que puede ser corroborado en los difractogramas de rayos-X. En la Figura 3 se presentan los difractogramas de rayos-X para el sistema dopado con bario. En la Figura 3a se presentan los difractogramas obtenidos para las diferentes estequiometrias de polvos cerámicos calcinados a $800{ }^{\circ} \mathrm{C} / 2 \mathrm{~h}$ del sistema $\mathrm{Bi}_{1-\mathrm{x}} \mathrm{Ba}_{\mathrm{x}} \mathrm{FeO}_{3}(\mathrm{x}=0.10,0.15$ y 0.20), en ella se puede apreciar que la fase mayoritaria es la fase correspondiente al BFO (JCDPS 72-2493), fase romboédrica y grupo espacial R3c; esta fase coexiste con un porcentaje mínimo de la fase $\mathrm{Bi}_{25} \mathrm{FeO}_{40} \cdot{ }^{28} \mathrm{Un}$ análisis más detallado del pico de mayor intensidad es presentado en la Figura 3(b), que corresponde a una ampliación del difractograma en el rango $31^{\circ}<2 \theta<33^{\circ}$, donde se ubican los picos (104) y (110); en ella se observa que este pico combinado, se desplaza a valores mayores de $2 \theta$ para el BFO dopado con $10 \%$ y $15 \%$ de bario, y para valores menores cuando se dopa con un $20 \%$, el desplazamiento a valores menores de $2 \theta$ indica un incremento en los parámetros de red, es decir, la sustitución con bario conduce a un incremento en la celda unitaria, puesto que el radio del $\mathrm{Ba}^{+2}(0,142 \mathrm{~nm})$ es mayor que el del $\mathrm{Bi}^{+3}(0,117) .{ }^{29}$ La separación entre los picos de difracción (104) y (110) se reduce con la sustitución de bario, lo que implica que la estructura romboédrica experimenta un cambio de fase a monoclínica, tetragonal, ${ }^{30,31}$ o trigonal $(\mathrm{R} 3 \mathrm{~m}){ }^{32}$

Algunos autores ${ }^{4}$ reportan que la sustitución de Bi por Ba en el BFO afecta la estructura generando una transformación de fase de romboédrica $(\mathrm{x}=0,05 ; 0,10 ; 0,15)$ a tetragonal $(\mathrm{x}=0,20 ; 0,25$; 0,30 ); la distorsión tetragonal (c/a) se reduce cuando aumenta el contenido de $\mathrm{Ba}$. Otros investigadores ${ }^{33}$ han encontrado que la incorporación de bario genera una transformación de fase de romboédrica a ortorrómbica y con el aumento de dopante sufre una nueva transformación a tetragonal, siendo que el grupo espacial correspondiente a la fase ortorrómbica varia con el contenido de dopante, asociándose el grupo espacial $\mathrm{Pbcm}$, para valores menores de dopaje, y al grupo espacial P222, cuando el porcentaje de este aumenta. ${ }^{33}$ Los difractogramas de rayos $\mathrm{X}$ de la Figura 4 corresponden a polvos cerámicos del sistema $\mathrm{BiFe}_{1-\mathrm{y}} \mathrm{Nb}_{\mathrm{y}} \mathrm{O}_{3}(\mathrm{y}=0,03 ; 0,06$ y 0,09 ), sometidas a tratamiento térmico de $800{ }^{\circ} \mathrm{C} / 2 \mathrm{~h}$. Todos los difractogramas presentados en la Figura 4 indican que la principal fase cristalina es la correspondiente al BFO, también se presenta un mínimo porcentaje de la fase espuria $\mathrm{Bi}_{25} \mathrm{FeO}_{40}$; los resultados indican que un contenido relativamente bajo de $\mathrm{Nb}^{5+}$ en la estructura $\mathrm{BFO}$ favorece la reducción de las fases espurias. Para investigar más a fondo las influencias del $\mathrm{Nb}^{5+}$ en la estructura del $\mathrm{BiFe}_{1}$. ${ }_{x} \mathrm{Nb}_{\mathrm{x}} \mathrm{O}_{3}$, se realizó una ampliación de los patrones de difracción en el rango de $2 \theta$ entre $31-33^{\circ}$ (Figura $4 \mathrm{~b}$ ), donde se ve claramente que cuando el valor de $\mathrm{x}$ aumenta, los picos de difracción se desplazan gradualmente a ángulo mayores, para $\mathrm{x}<0,20$, y a ángulos menores, para $x \geq 0,20$, lo que indica una disminución gradual de los parámetros de red para $\mathrm{x}<0,20 \mathrm{y}$ un aumento de estos parámetros para $x \geq 0,20$. El cambio en los parámetros de red hasta $x=0,20$ se podría justificar considerando la distorsión de la red debido a la variación del contenido de $\mathrm{Nb}^{5+}$, ya que los radios iónicos del $\mathrm{Nb}^{5+}$ y $\mathrm{Fe}^{3+}$, en coordinación 6 son diferentes y para composiciones con $\mathrm{x} \geq 0,20$, el cambio en los parámetros de red y el desplazamiento de los picos hacia ángulos de Bragg menores puede ser debido a la presencia de fases espurias. ${ }^{34}$

En la Figura 5 se presentan los difractogramas de los polvos cerámicos del sistema $\mathrm{Bi}_{1-\mathrm{x}} \mathrm{Ba}_{\mathrm{x}} \mathrm{Fe}_{1-\mathrm{y}} \mathrm{Nb}_{\mathrm{y}} \mathrm{O}_{3}$ para una concentración de $10 \%$ de bario y diferentes concentraciones de niobio $(\mathrm{y}=0,03 ; 0,06 \mathrm{y}$ $0,09)$. Se observa claramente que utilizando este método de síntesis y además con esta concentración de dopantes, los difractogramas muestran una evidente eliminación de las fases secundarias presentes en las muestras para las diferentes concentraciones de niobio. El pequeño cambio en los picos de difracción a un ángulo mayor sugiere una ligera reducción en los parámetros de red; la incorporación de un ion de menor tamaño en la estructura daría lugar a la contracción en la celda unidad. ${ }^{35}$ En la Figura 5(b) claramente se observa que los picos (104) y (110) tienden a formar un solo pico en las muestras co-dopadas con $\mathrm{Ba}-\mathrm{Nb}$, lo que indica una disminución en el grado de distorsión estructural del BFO y la estructura romboédrica puede cambiar ligeramente a pseudocúbica. ${ }^{36}$

El ancho de línea de los picos correspondientes a los patrones de difracción son el resultado de dos contribuciones: la de tamaño del cristalito $\beta_{t}$ y la causada por la deformación experimentada por la deformación de la red $\beta_{d}$, y puede ser expresada como ${ }^{37}$
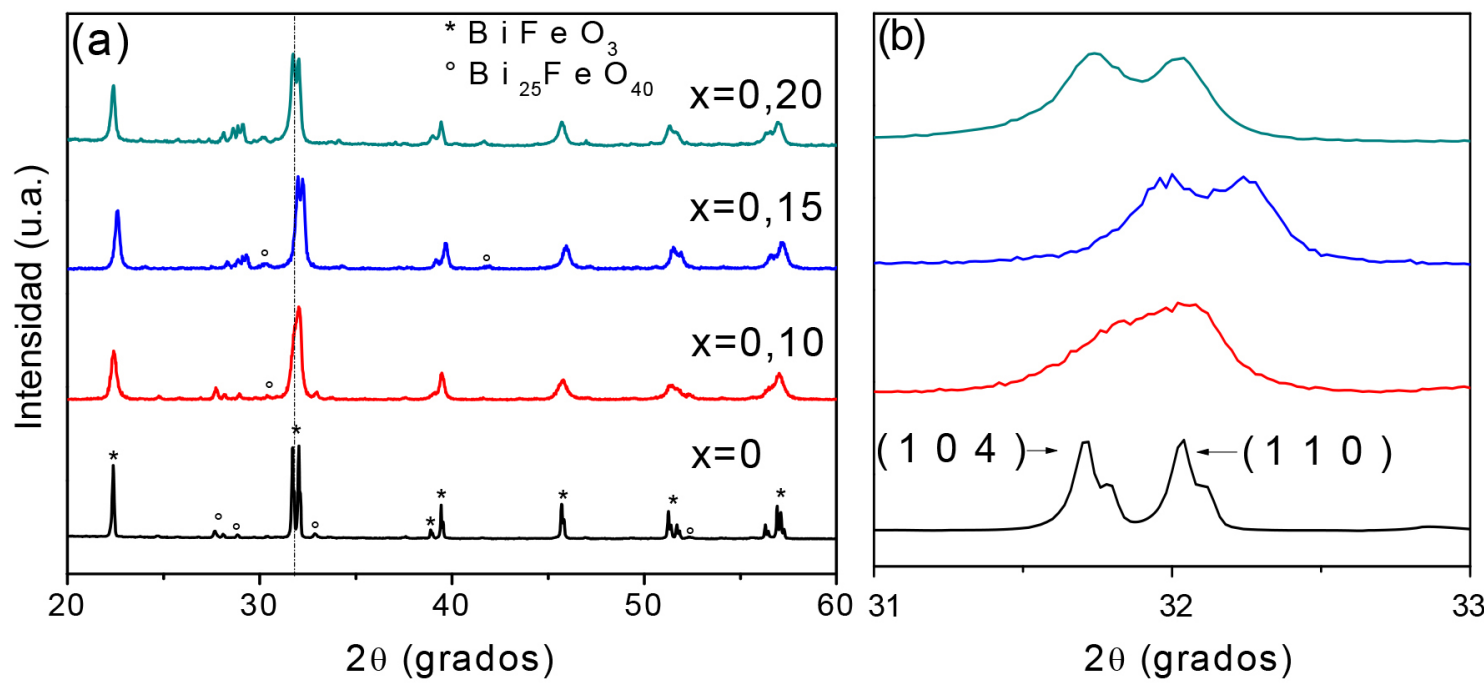

Figura 3. Difractogramas de los polvos cerámicos del sistema $\mathrm{Bi}_{1-x} \mathrm{Ba}_{x} \mathrm{Fe} \mathrm{O}_{3}$, para diferentes concentraciones de bario sintetizados por el método de Gel citrato 


$$
\beta=\beta_{t}+\beta_{d}
$$

Donde la contribución del tamaño del cristalito a la amplitud del pico de difracción, está dada por la ecuación de Scherrer $\beta_{t}=\frac{0.94 \lambda}{D \cos \theta}$, donde $\mathrm{D}$ es el tamaño promedio del cristalito, $\lambda$ es la longitud de onda de los rayos-X $(\lambda=1.5406 \AA) .{ }^{38}$ En un cristal distorsionado el espaciamiento interplanar $d$ no es constante y distintas partes del cristal difractarán ángulos diferentes, la contribución al ancho de línea puede ser calculado de la relación de Bragg, $n \lambda=2 d \operatorname{sen} \theta$. Si $\varepsilon$ es la deformación máxima que experimenta el cristal, el espaciamiento interplanar variará de $d(1+\varepsilon)$ a $d(1-\varepsilon)$ y $\theta$ variará sobre un rango numérico igual a $2 \varepsilon d\left(\frac{\partial \theta}{\partial d}\right)$, o $2 \varepsilon \tan \theta$, puesto que el patrón de difracción está en función de $2 \theta$, el rango angular sobre el cual la reflexión es apreciable será dos veces este valor, o sea, $\beta_{d}=4 \varepsilon \tan \theta$, donde $\varepsilon$ es la deformación de la red. ${ }^{39}$

$$
\begin{gathered}
\beta=\frac{0.94 \lambda}{D \cos \theta}+4 \varepsilon \tan \theta \\
\beta \cos \theta=\frac{0.94 \lambda}{D}+4 \varepsilon \operatorname{sen} \theta
\end{gathered}
$$

Las anteriores expresiones son las ecuaciones $\mathrm{W}-\mathrm{H}$, graficando entonces $\beta \cos \theta$ vs $4 \operatorname{sen} \theta$ (Figura 6), podemos encontrar el tamaño del cristalito del intercepto con el eje y, del valor de la pendiente se obtiene la deformación, los resultados se presentan en la Tabla 1.

Los polvos cerámicos del sistema $\mathrm{Bi}_{1-x} \mathrm{Ba}_{x} \mathrm{FeO}_{3}$ calcinados a $800{ }^{\circ} \mathrm{C} / 2 \mathrm{~h}$, se caracterizaron por microscopia electrónica de barrido (MEB) para establecer la morfología y el tamaño de partícula; las micrografías del material se muestran en las Figuras 7 para las composiciones (a) $\mathrm{x}=0 \mathrm{y}$ (b) $\mathrm{x}=0.10$.

De manera general, las micrografías permiten apreciar que las partículas correspondientes a la muestra sin dopar (7a) presentan forma irregular y tamaño micrométrico. Con el dopaje se logró reducir considerablemente el tamaño de partícula, lo que está de acuerdo con los cálculos consignados en la Tabla 1, obteniéndose partículas de tamaño nanométrico que varian entre $309 \mathrm{~nm}$ a $65 \mathrm{~nm}$, dependiendo del porcentaje del dopante, teniendo todas forma irregular (7b). Para la muestra de BFO sin dopar se observó la presencia de aglomerados, formados por partículas con forma de dodecaedros regulares; los aglomerados se eliminaron sustancialmente con el dopaje. El proceso de aglomeración puede ser atribuido a la reducción de la energía superficial, las partículas primarias tienen una tendencia a formar aglomerados casi esféricos en una razón mínima de superficie/
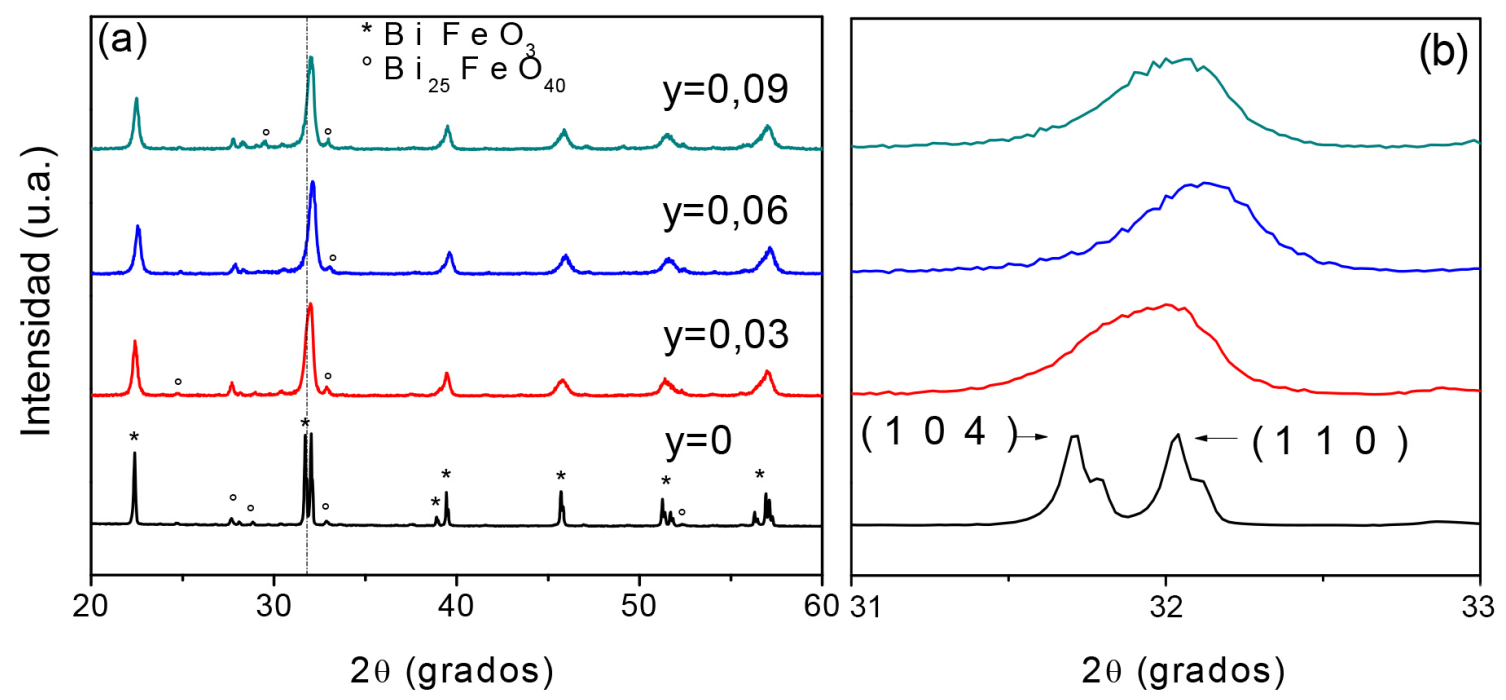

Figura 4. Difractogramas de los polvos cerámicos del sistema BiFe $e_{1-y} \mathrm{Nb}_{y} \mathrm{O}_{3}$, para diferentes concentraciones de Niobio, sintetizados por el método de Gel citrato
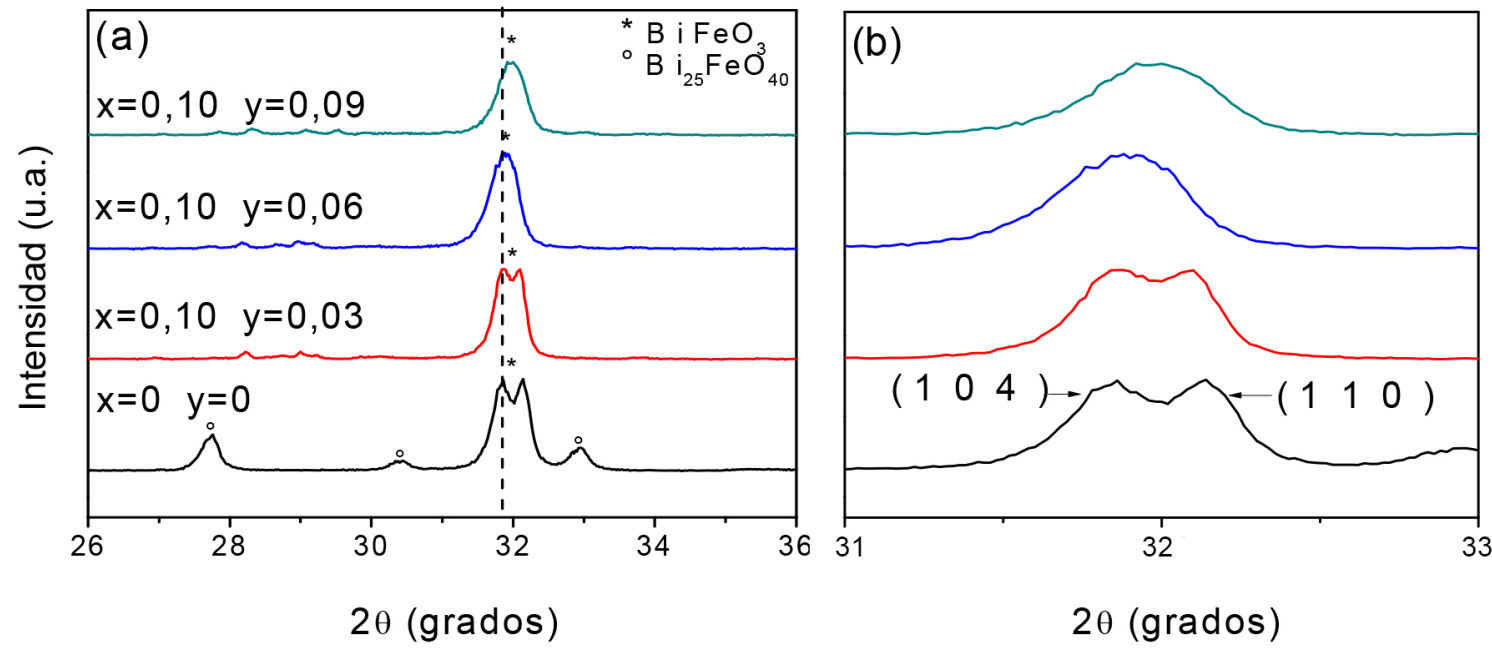

Figura 5. Difractogramas de los polvos cerámicos del sistema $\mathrm{Bi}_{0,90} \mathrm{Ba}_{0,10} \mathrm{Fe}_{1-\mathrm{y}} \mathrm{Nb}_{\mathrm{y}} \mathrm{O}_{3}$, para diferentes concentraciones de Niobio, sintetizados por el método de Gel citrato 

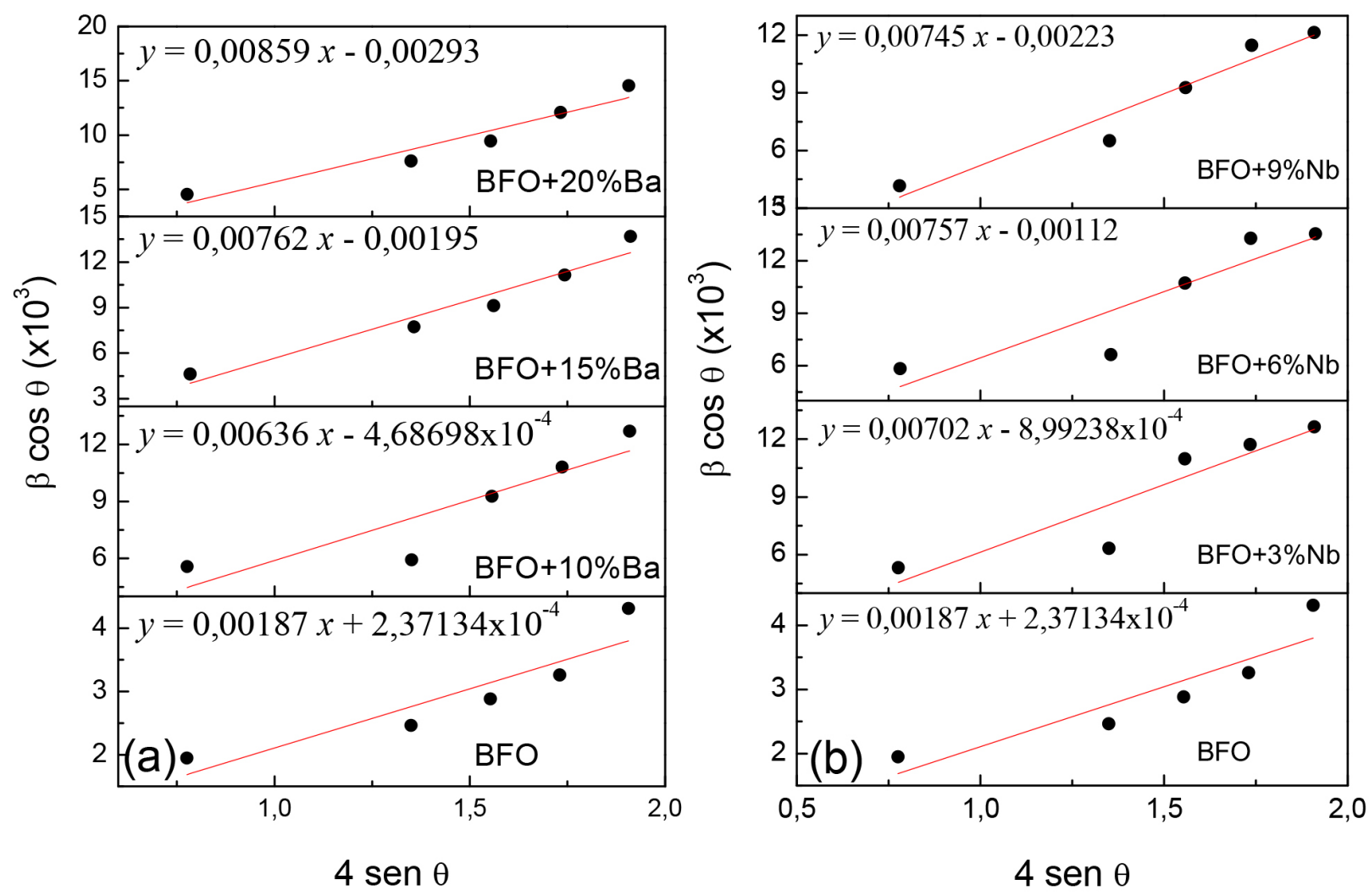

Figura 6. Variación de $\beta \cos \theta$ vs $4 \operatorname{sen} \theta$ para los sistemas (a) $\mathrm{Bi}_{1-x} \mathrm{Ba} a_{x} \mathrm{FeO}_{3} y$ (b) $\mathrm{BiFe}_{1-y} \mathrm{Nb}_{y} \mathrm{O}_{3}$

Tabla 1. Parámetros de red y propiedades del sistema $\mathrm{Bi}_{1-\mathrm{X}} \mathrm{Ba}_{\mathrm{X}} \mathrm{Fe}_{1-\mathrm{y}} \mathrm{Nb}_{\mathrm{y}} \mathrm{O}_{3}$

\begin{tabular}{|c|c|c|c|c|c|}
\hline \multirow{2}{*}{ Composición } & \multicolumn{2}{|c|}{ Parámetros de red } & \multirow{2}{*}{ Volumen } & \multicolumn{2}{|c|}{ W-H } \\
\hline & $a$ & $c$ & & Deformación (\%) & Tamaño cristalito $(\mathrm{nm})$ \\
\hline $\mathrm{BFO}$ & 5,571 & 1,612 & 144,49 & 0,19 & 611 \\
\hline BFO-10Ba & 5,555 & 1,618 & 144,12 & 0,64 & 309 \\
\hline BFO-15Ba & 5,610 & 1,545 & 140,37 & 0,76 & 74 \\
\hline BFO-20Ba & 5,551 & 1,625 & 144,53 & 0,86 & 49 \\
\hline $\mathrm{BFO}-3 \mathrm{Nb}$ & 5,555 & 1,618 & 144,13 & 0,70 & 161 \\
\hline $\mathrm{BFO}-6 \mathrm{Nb}$ & 5,611 & 1,549 & 140,73 & 0,76 & 129 \\
\hline BFO-9Nb & 5,578 & 1,587 & 142,53 & 0,75 & 65 \\
\hline
\end{tabular}

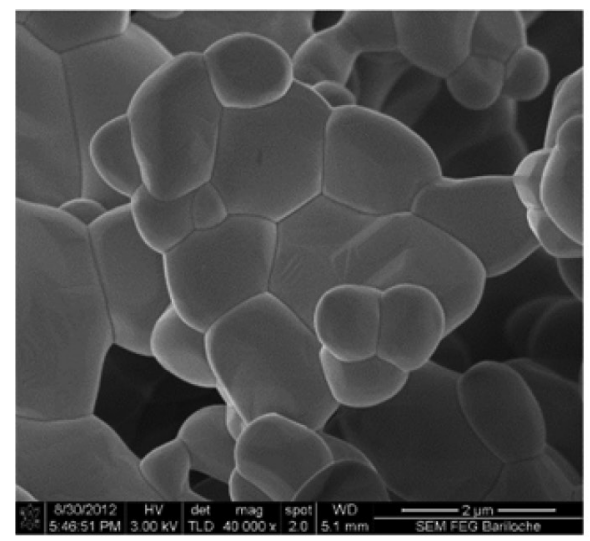

(a)

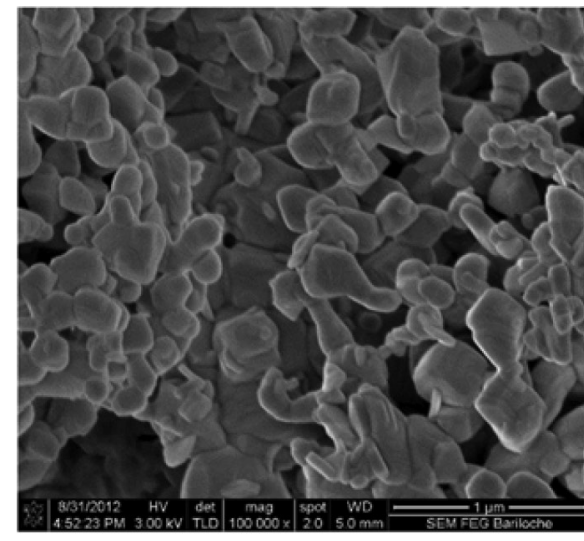

(b)

Figura 7. Microscopia electrónica de barrido del sistema $\mathrm{Bi}_{l_{-x}} \mathrm{Ba}_{x} \mathrm{Fe} \mathrm{O}_{3}$, con (a) $x=0$, (b) $x=0.10$ 
volumen; con esta disposición se logra reducir la energía libre superficial, este tipo de estructura es común en óxidos, ferritas y titanatos. ${ }^{40}$ En las Figuras $8(a, b, c)$ se presenta la relación de la parte real de la impedancia, esto es la resistencia, vs frecuencia para un amplio rango de temperaturas para los sistemas (a) $\mathrm{BiFeO}_{3}$ sin dopar, (b) $\mathrm{BiFe}_{0.94} \mathrm{Nb}_{0.06} \mathrm{O}_{3}$ y (c) $\mathrm{Bi}_{0.90} \mathrm{Ba}_{0.10} \mathrm{Fe}_{0.94} \mathrm{Nb}_{0.06} \mathrm{O}_{3}$. Se observa que $\mathrm{Z}^{\prime}$ decrece abruptamente con el aumento de la frecuencia hasta un valor de $\sim 10 \mathrm{kHz}$ y luego tiende a un valor constante lo que indica la presencia de relajación dieléctrica en estos compuestos. ${ }^{41}$ La fusión de las curvas de $Z^{\prime}$ para altas frecuencias, puede ser debida a la liberación de cargas espaciales como resultado de la reducción en las propiedades de barrera del material con el aumento de la temperatura y puede ser un factor responsable del aumento de la conductividad en el material con el aumento de la temperatura y la frecuencia. ${ }^{42}$ Además, a bajas frecuencias se observa el decremento en el valor de $Z^{\prime}$ con el aumento de la temperatura lo que sugiere una resistencia dependiente de la temperatura con coeficiente de temperatura negativo (NTC) similar al de los materiales semiconductores. ${ }^{43}$ En las Figuras 8(d, e, f) se presenta la relación de la parte imaginaria de la impedancia vs frecuencia para un amplio rango de temperaturas para los sistemas (d) $\mathrm{BiFeO}_{3}$ sin dopar, (e) $\mathrm{BiFe}_{0.94} \mathrm{Nb}_{0.06} \mathrm{O}_{3}$ y (f) $\mathrm{Bi}_{0.90} \mathrm{Ba}_{0.10} \mathrm{Fe}_{0.94} \mathrm{Nb}_{0.06} \mathrm{O}_{3}$. Los gráficos de $Z^{\prime \prime}$ vs frecuencia se caracterizan por (i) la aparición de un pico con un valor máximo a una cierta frecuencia, (ii) ancho asimétrico de los picos, (iii) el desplazamiento del valor de Z" máx a frecuencias más altas con el aumento de la temperatura y (iv) disminución en la magnitud de Z" ${ }_{\text {max }}$ con el aumento de la temperatura. Los fenómenos de relajación hacen referencia al acercamiento de un sistema a una nueva condición de equilibrio, o de estado estacionario. El proceso de relajación puede ser debido a la presencia de electrones/especies inmóviles a bajas temperaturas y a defectos/vacancias a temperaturas más altas. ${ }^{44}$ La altura de los picos es proporcional a la resistencia del bulk $\left(\mathrm{R}_{\mathrm{b}}\right)$, y puede ser estimada y explicada por la expresión $Z^{\prime \prime}=\left[R_{b}\left\{\frac{\omega \tau}{1+\omega^{2} \tau^{2}}\right\}\right]{ }^{44}$ Además, la magnitud de $Z^{\prime \prime}$ disminuye gradualmente con un cambio en la frecuencia del pico hacia el lado de altas frecuencias y finalmente las curvas se funden en este rango de frecuencias, esto indica la acumulación de cargas espaciales en los materiales, ${ }^{44} \mathrm{el}$ ancho asimétrico sugiere que hay una distribución en los valores de los tiempos de relajación. Además, el cambio de valor $\mathrm{Z}^{\prime \prime}{ }_{\max }$ al lado de frecuencias más altas, con la temperatura, indica la existencia de un fenómeno de relajación eléctrica en el material dependiente de la temperatura. ${ }^{45} \mathrm{La}$ disminución del valor máximo $\mathrm{Z}^{\prime \prime}{ }_{\text {max }}$ en cada una de las curvas con el aumento de la temperatura denota la disminución de la resistencia y el aumento en las pérdidas del material, puesto que $Z^{\prime \prime}{ }_{\text {max }}=\mathrm{R} / 2 \cdot{ }^{46,47} \mathrm{El}$ ensanchamiento del pico con el aumento de la temperatura sugiere la presencia de un fenómeno de relajación eléctrico dependiente de la temperatura. ${ }^{45}$ Los grandes valores de $Z^{\prime}$ y $Z^{\prime \prime}$ para los menores valores de frecuencias y temperaturas indican mayores efectos de la polarización a baja temperatura donde la conductividad es baja y la impedancia es alta. A bajas temperaturas las especies de relajación son especies inmóviles como se indica por la caída monótona de Z" con la frecuencia lo que indica que predomina el efecto orientacional. ${ }^{48}$ Las medidas de magnetización vs campo magnético fueron realizadas a temperatura ambiente $(\sim 298 \mathrm{~K})$ con un barrido entre -40 y $40 \mathrm{kOe}$, para todos los sistemas estudiados.

En la Figura 9(a) se puede observar la curva de histéresis obtenida para la muestra de $\mathrm{BiFeO}_{3}$ sin dopar, la parte ampliada de la curva M-H obtenida permite observar la no-linealidad de ésta, evidenciando una magnetización remanente de aproximadamente 0,005 emu/g y un campo coercitivo de $\sim 152 \mathrm{Oe}$, datos que concuerdan con lo reportado en la literatura, ${ }^{49}$ evidenciándose ferromagnetismo débil a temperatura ambiente, lo que se confirma con los valores de coercitividad distintos de cero. Este ferromagnetismo débil se debe principalmente a la distribución de iones $\mathrm{Fe}^{2+}$ y $\mathrm{Fe}^{3+}$ existentes para lograr la compensación de cargas generadas por la presencia de vacancias de oxígeno y la interacción magnética entre ellos conduce a la magnetización espontánea observada. ${ }^{50}$ Se conoce que el BFO muestra un ordenamiento antiferromagnético tipo $\mathrm{G}$, pero tiene un momento magnético residual causado por su estructura "inclinada" de espines (ferromagnetismo débil). El orden ferromagnético débil en sí se puede entender también como un resultado de los arreglos de espines no-colineales (inclinados) en dos subredes. ${ }^{49}$ En la Figura 9 (b, c, d) se observan las curvas de histéresis para las muestras que contienen los tres porcentajes de niobio utilizados como dopaje (3\%, $6 \%$ y $9 \%$ ), tal que todas las muestras dopadas con $\mathrm{Nb}^{5+}$ exhibieron ciclos de histéresis magnética, correspondiente a un comportamiento ferromagnético. La aparición de ferromagnetismo en el BFO dopado con niobio, puede ser atribuido a la inclinación de los espines ordenados antiferromagnéticamente debido a una distorsión estructural o a la ruptura del equilibrio en la magnetización (generada por el $\mathrm{Fe}^{3+}$ ) de las subredes antiparalelas debido a la sustitución del ión metálico por otro de diferente valencia. ${ }^{5}$ Para la muestra dopada con 3\% de niobio, la magnetización remanente $\left(\mathrm{M}_{\mathrm{r}}=0,044 \mathrm{emu} \mathrm{\textrm {g } ^ { - 1 }}\right)$ y la magnetización de saturación $\left(\mathrm{M}_{\mathrm{s}}=0,307 \mathrm{emu} \mathrm{g}^{-1}\right)$ aumentan significativamente en comparación con los valores de BFO sin dopar. Para un dopaje con $6 \%$ los valores para el campo coercitivo, magnetización remanente y magnetización de saturación fueron:

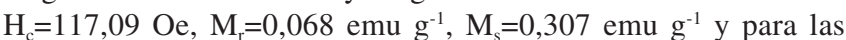
dopadas con $9 \%$ de niobio estos valores fueron: $\mathrm{H}_{\mathrm{c}}=5,04 \mathrm{kOe}$, $M_{r}=0,019 \mathrm{emu} \mathrm{g}^{-1}, M_{\mathrm{s}}=0,1754 \mathrm{emu} / \mathrm{g}$, respectivamente. Cabe señalar que el $\mathrm{Nb}^{5+}$ no es un ión magnético así que el dopaje con $\mathrm{Nb}^{5+}$ no contribuye directamente al ferromagnetismo. El ferromagnetismo observado sólo puede provenir de la interacción $\mathrm{Fe}^{3+}-\mathrm{O}^{2-}-\mathrm{Fe}^{3+}$ de superintercambio. ${ }^{51}$ Es importante anotar que las fases espurias presentes en las muestras no tienen propiedades magnéticas por tanto no contribuyen a la respuesta magnética observada, así que la respuesta ferromagnética puede atribuirse a la destrucción de la modulación cicloidal y la inclinación de la estructura de espines antiferromagnética. ${ }^{51}$ La mejora de la magnetización, a temperatura ambiente, en el sistema dopado puede ser explicado como resultado de la inserción del $\mathrm{Nb}^{5+}$ en la subred de Fe. Aunque los iones de $\mathrm{Nb}^{5+}$ no son magnéticamente activos, y no pueden contribuir directamente a la magnetización, pueden perturbar la modulación espacial de los espines y destruir así el acoplamiento cicloidal, mediante la sustitución de algunos iones $\mathrm{Fe}^{3+}$ magnéticamente activos. Además, si tenemos en cuenta que el ordenamiento antiferromagnético en el BFO sin dopar es derivado de la interacción de super-intercambio a través de los iones de oxígeno, el reemplazar algunos iones de $\mathrm{Fe}^{3+}$ por iones de $\mathrm{Nb}^{5+}$ permitirá un acoplamiento ferromagnético en lugar de uno antiferromagnético.

Por lo tanto, sobre la base de este modelo, un mayor ordenamiento entre $\mathrm{Fe}^{3+} \mathrm{y} \mathrm{Nb}^{5+}$ dará como resultado un valor mayor de magnetización. ${ }^{52}$ En la Figura 10 se presentan las curvas de magnetización en función del campo aplicado (a temperatura ambiente) para las muestras dopadas simultáneamente con bario y niobio, manteniendo constante el contenido de bario $(\mathrm{x}=0,10)$ y variando el porcentaje de niobio (3\%, 6\% y 9\%). Los valores de campo coercitivo, magnetización remanente y magnetización de saturación obtenidos para el sistema $\mathrm{Bi}_{09} \mathrm{Ba}_{01} \mathrm{Fe}_{1-\mathrm{y}} \mathrm{Nb}_{\mathrm{y}} \mathrm{O}_{3}$, con $\mathrm{y}=0,03$, fueron, $\mathrm{H}_{\mathrm{c}}=3,66 \mathrm{kOe}, \mathrm{M}_{\mathrm{s}}=$ $0,863 \mathrm{emu} \mathrm{g}^{-1} \mathrm{y} \mathrm{Mr}=0,41 \mathrm{emu} \mathrm{g}^{-1}$. Cuando el porcentaje de niobio se incrementó a un $6 \%$, estos valores cambiaron a $\mathrm{H}_{\mathrm{c}}=2,97 \mathrm{kOe}, \mathrm{M}_{\mathrm{s}}=$

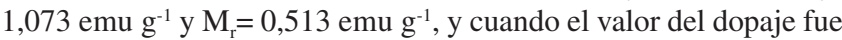
de $9 \%, \mathrm{H}_{\mathrm{c}}=3,04 \mathrm{kOe}, \mathrm{M}_{\mathrm{s}}=0,413 \mathrm{emu} \mathrm{g}^{-1}$ y $_{\mathrm{r}}=0,177 \mathrm{emu} \mathrm{g} \mathrm{g}^{-1}$, la respuesta magnética pudo ser manipulada controlando el contenido de bario. ${ }^{4}$ Ya que en las muestras se mantuvo constante el contenido de 

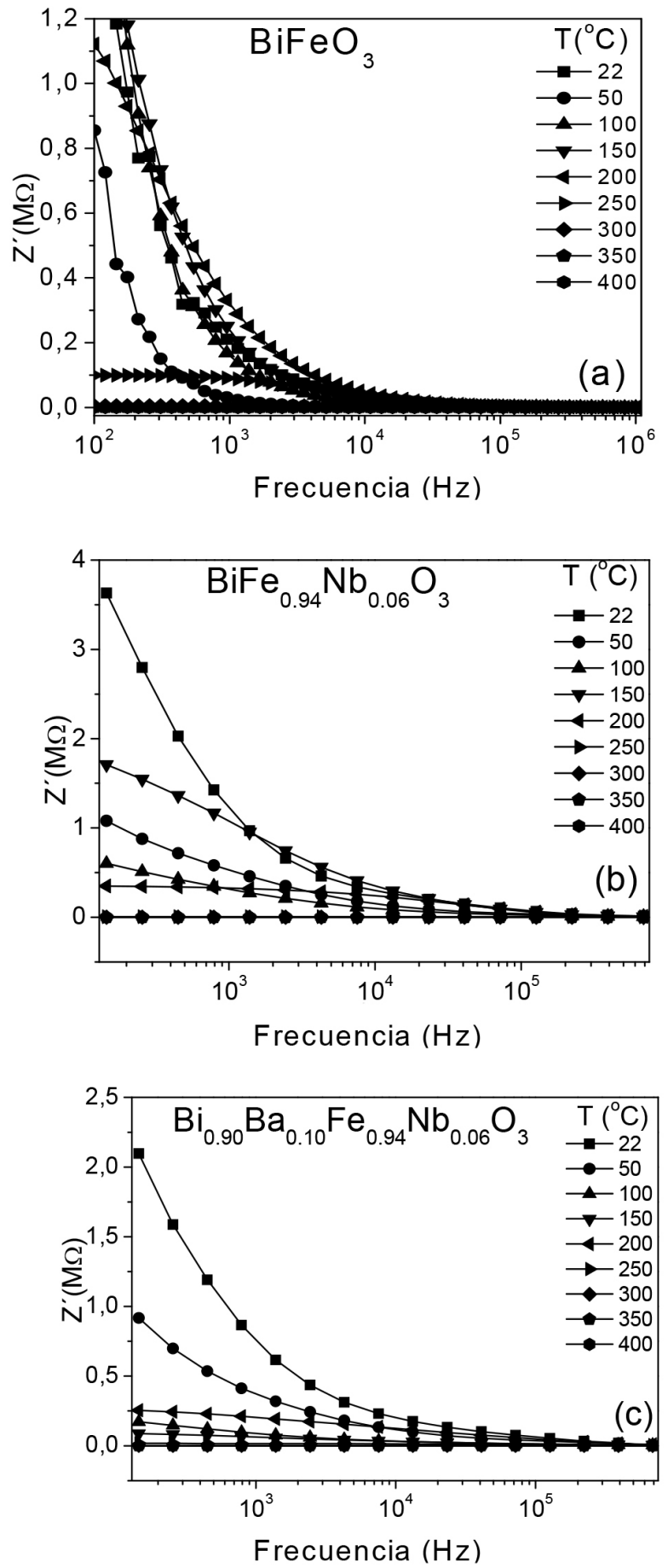
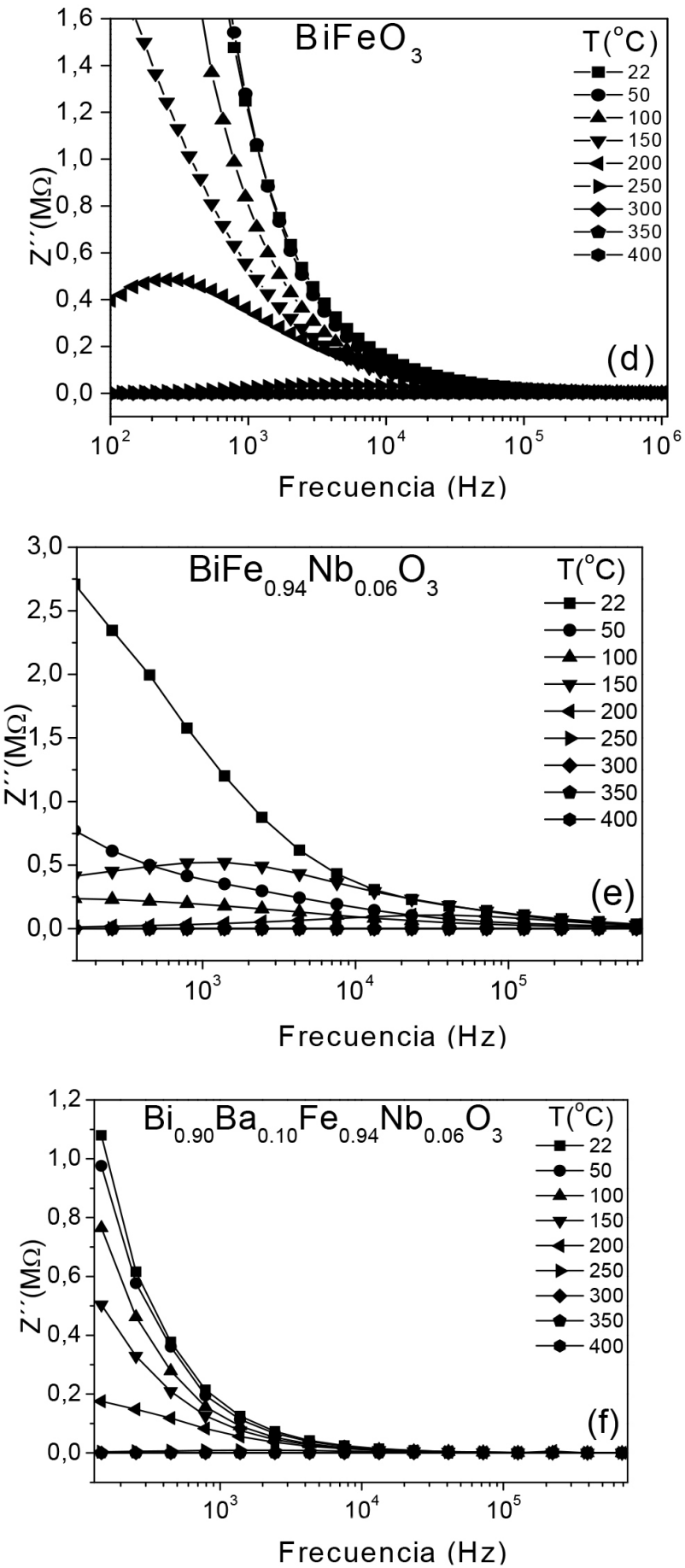

Figura 8. Espectros de impedancia $Z^{\prime}$ vs. frecuencia $(a, b, c)$ y $Z^{\prime \prime}$ vs. frecuencia $(d$, e, $f)$ correspondientes a los sistemas (a, d) BiFeO ${ }_{3}$ sin dopar, (b, e) Bi$\mathrm{Fe}_{0.94} \mathrm{Nb}_{0.06} \mathrm{O}_{3} y(c, f) \mathrm{Bi}_{0.90} \mathrm{Ba}_{0.10} \mathrm{Fe}_{0.94} \mathrm{Nb}_{0.06} \mathrm{O}_{3}$

bario, se observó que la respuesta magnética obtenida fue fuertemente dependiente del contenido de niobio.

Comparando los valores anteriores con los obtenidos para el sistema $\mathrm{BiFe}_{1-\mathrm{y}} \mathrm{Nb}_{\mathrm{y}} \mathrm{O}_{3}$, considerando los mismos porcentajes de niobio, en general, los valores de los parámetros antes mencionados aumentaron. Los valores de campo coercitivo para las muestras co-dopadas con $3 \%$ y $6 \%$ aumentaron notablemente, sin embargo, para la muestra que posee el mayor porcentaje de niobio, este disminuyó en comparación con el de la muestra dopada solo con niobio. Del análisis realizado con difracción de rayos $\mathrm{X}$ se concluyó que las muestras dopadas sólo con niobio experimentaron simultáneamente, una transición de fase y contracción de la red. Mientras que en las muestras co-dopadas (10\% de Ba y $3 \%$ - $6 \%$ de $\mathrm{Nb}$ ), sólo se observó un aumento en los parámetros de red y la transición de fase sólo tuvo lugar cuando se incorporó 9\% de niobio en la estructura. Por tanto, el cambio en los valores de los parámetros magnéticos puede ser ocasionado por el cambio en la estructura cristalina. El origen de la magnetización espontánea, asociada con el bario, se puede explicar de la siguiente manera: en primer lugar, la sustitución del bismuto (trivalente) por un ión divalente requiere de una compensación de carga, que se consigue con la creación de vacancias de oxígeno; ${ }^{4,53}$ la deficiencia de oxígeno se incrementó cuando el porcentaje de bario aumentó. En 

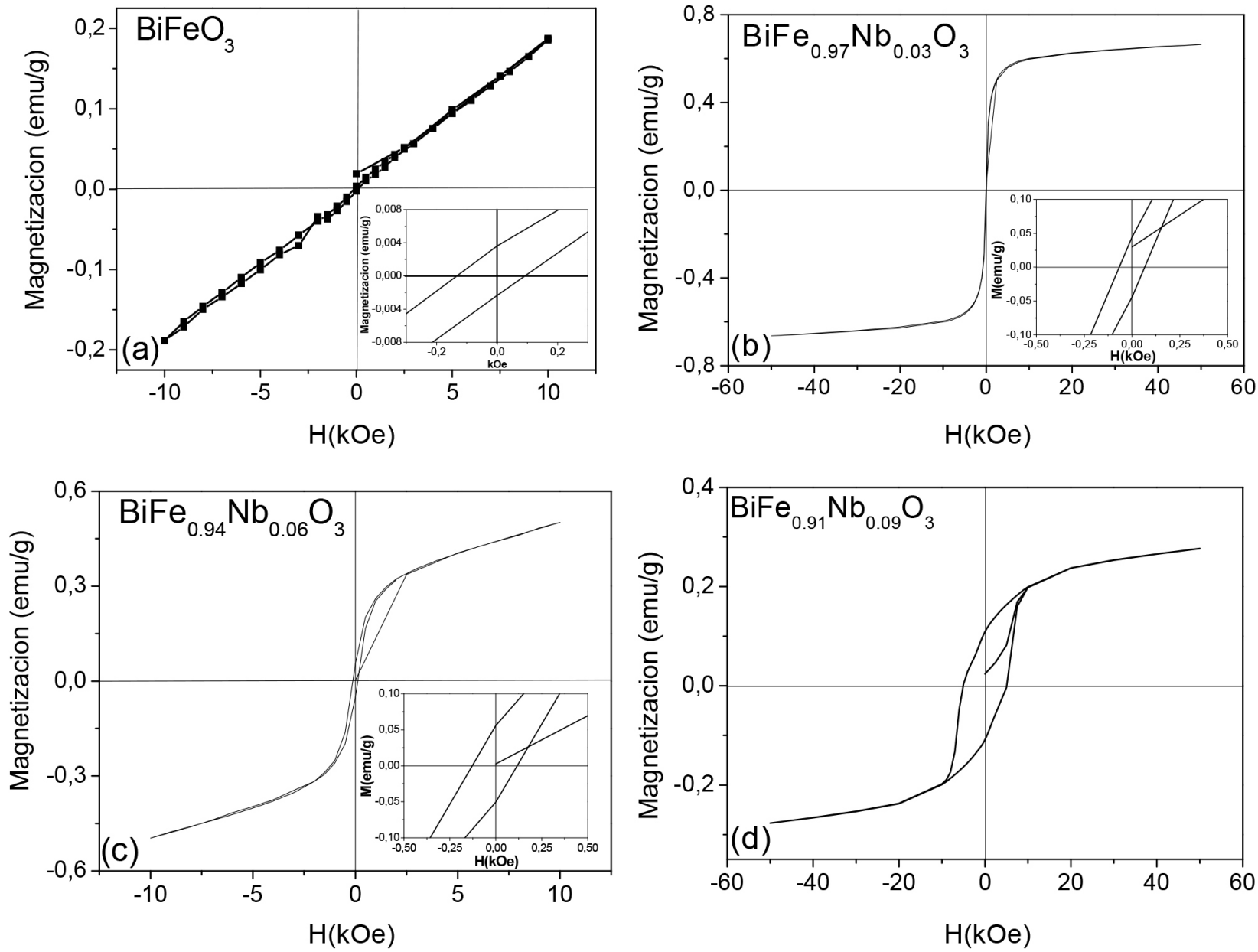

Figura 9. Curva de histéresis de las muestras del sistema BiFe ${ }_{1-y} \mathrm{Nb}_{y} \mathrm{O}_{3}$ a distintas concentraciones de niobio (a) y=0, (b) y=0.03, (c) y=0.06 y (d) y=0.09

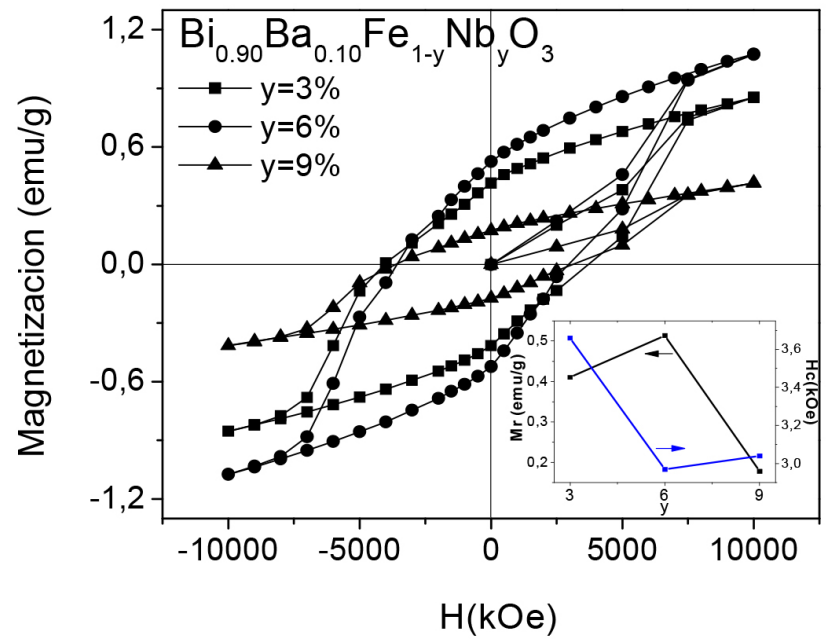

Figura 10. Curva de histéresis de las muestras del sistema $\mathrm{Bi}_{0.90} \mathrm{Ba} a_{0.10} \mathrm{Fe}_{1-y} \mathrm{Nb}_{y} \mathrm{O}_{3}$

segundo lugar, el BFO con una estructura tipo perovskita romboédrica distorsionada permitió una respuesta ferromagnética débil debido a la inclinación de los espines. Cuando la estructura se dopó con bario, la estructura romboédrica cambió a ortorrómbica modificándose el ángulo del enlace $\mathrm{Fe}-\mathrm{O}-\mathrm{Fe}$, lo que generó una magnetización neta; en conclusión, dopando el BFO con bario la respuesta magnética mejoró notablemente.

\section{CONCLUSIONES}

El método Gel citrato permitió la obtención de la fase perovskita para el sistema de interés en todas las estequiometrias estudiadas, esto evidencia que la homogeneidad a nivel molecular alcanzada en este método favorece la formación de la fase deseada, obteniéndose partículas irregulares y de tamaño nanométrico; la variación en la estequiometria afecta los enlaces presentes en la estructura perovskita, lo que se puede apreciar a través del análisis realizado a los espectros infrarrojos. La difracción de rayos-X permitió apreciar un ligero corrimiento en los picos de difracción como resultado de la diferencia de tamaño de los cationes. La inclusión de bario en la estructura cristalina del $\mathrm{BiFeO}_{3}$ genera simultáneamente una distorsión de la red cristalina y una transformación de fase, lo que se ve reflejado en la respuesta ferromagnética del sistema a temperatura ambiente, esta respuesta se debe posiblemente a la influencia que posee el dopaje en el ordenamiento de espines, lo que puede generarse por la creación de vacancias de oxígeno al dopar con un ion de valencia diferente. La inclusión de niobio en la estructura mejora notablemente la respuesta magnética del material debido a la ruptura en la alineación de espines ya que el niobio ocupa algunos de los octaedros ocupados por el hierro, esta "ruptura" genera la respuesta magnética de la muestra dopada, adicionalmente el niobio tiene una valencia diferente al catión que reemplaza, haciendo que la compensación de carga favorezca la respuesta magnética del material, haciendo que el material obtenga una respuesta ferromagnética a temperatura ambiente. Los resultados obtenidos del dopaje simultáneo nos llevan a concluir que el dopaje con grandes porcentajes de bario afecta desfavorablemente 
la respuesta magnética y pequeños porcentajes de niobio también favorecen el comportamiento ferromagnético, por tanto se podrían emplear pequeños porcentajes de los dos dopantes para favorecer el comportamiento ferromagnético. Los sistemas estudiados presentan comportamiento ferroeléctrico, reflejado en su respuesta dieléctrica y comportamiento ferromagnético reflejado en las curvas de histéresis magnética, obtenidas a temperatura ambiente. Los resultados obtenidos por el método de Williamson-Hall están de acuerdo con las micrografías y los patrones de difracción de rayos-X obtenidos.

\section{AGRADECIMENTOS}

Los autores agradecemos a la Universidad del Cauca por el apoyo brindado durante el desarrollo de esta investigación. y a la Companhia Brasileira de Metalurgia e Mineração (CBMM) por la donación del oxalato amoniacal de niobio utilizado en este estudio.

\section{REFERÊNCIAS}

1. Catalan, G.; Scott, J. F.; Adv. Mater. 2009, 21, 2463.

2. Zhan, S.-T.; Zhang, Y.; Lu, M.-H.; Du, C.-L.; Chen, Y.-F.; Liu, Z. G.; Zhu, Y.-Y.; Ming, N.-B.; Appl. Phys. Lett. 2006, 88, 162901.

3. Khomchenko, V. A.; Kiselev, D. A.; Kopcewicz, M.; Maglione, M.; Shvartsman, V. V.; Borisov, P.; Kleemann, W.; Lopes, A. M. L.; Pogorelov, Y. G.; Araujo, J. P.; Rubinger, R. M.; Sobolev, N. A.; Vieira, J. M.; Kholkin, A. L.; J. Magn. Magn. Mater. 2009, 321, 1692.

4. Yang, C.; Jiang, J.-S.; Qiana, F.-Z.; Jiang, D.-M.; Wang, C.-M.; Zhang, W.-G.; J. Alloys Compd. 2010, 507, 29.

5. Jun, Y.-K.; Moon, W.-T.; Chang, C.-M.; Kim, H.-S.; Ryu, H. S.; Kim, J. W.; Kim, K. H.; Hong, S.-H.; Solid State Commun. 2005, 135, 133.

6. Jun, Y-K.; Hong, S-H.; Solid State Commun. 2007, 144, 329.

7. Cullity, B. D.; Stock, S. R.; Elements of X-ray diffraction, Pearson Education Limited, USA, 2014.

8. Rietveld, H. M.; Acta Crystallogr. 1976, 22, 151.

9. Balzar, D.; Ledbetter, H.; J. Appl. Crystallogr. 1993, 26, 97.

10. Warren, B. E.; Averbach, B. L.; J. Appl. Phys. 1950, 21, 595.

11. Suryanarayana, C.; Grant Norton, M.; X-ray Diffraction: A Practical Approach. Springer, New York, 1998.

12. Piñeros, R. A.; Moreno Aldana, L. C.; Valencia Rios, J. S.; Revista Colombiana de Física 2006, 38, 1.

13. Wu, Z. J.; Zhao, X. B.; Tu, J.; Cao, G. S.; Tu, J. P.; Zhu, T. J.; J. Alloys Compd. 2005, 403, 345 .

14. Feldhoff, A.; Arnold, M.; Martynczuk, J.; Gesing, Th. M.; Wang, H.; Solid State Sci. 2008, 10, 689.

15. Nakamoto, K.; Infrared and Raman spectra of inorganic and coordination compounds Part B: applications in coordination, organometallic, and bioinorganic chemistry, $6^{\text {th }}$ ed., John Wiley \& Sons, Inc.: Hoboken, 2099.

16. Fernández-Perdomo, C. P.; Rivera, E.; Rodríguez-Páez, J. E.; Rev. Acad. Colomb. Cienc. Exactas, Fis. Nat. 2009, 33, 93.

17. Ardelean, I.; Cora, S.; Ioncu, V.; J. Optoelectron. Adv. Mat. 2006, 8, 1843.

18. Saeid, F.; Masoumeh, Z.; J. Mol. Catal. A: Chem. 2009, 299, 18.

19. Chen, Z.; He, X.; J. Alloys Compd. 2010, 97, 312.

20. Rao, T. D.; Karthik, T.; Srinivas, A.; Asthana, S.; Solid State Commun. 2012, 152, 2071.

21. Dunne, P. W.; Starkey, C. L.; Munn, A. S.; Tang, S. V. Y.; Luebben, O.; Shvets, I.; Ryder, A. G.; Casamayou-Boucau, Y.; Morrison, L.; Leste, E. H.; Chem. Eng. J. 2016, 289, 433.
22. Lanfredi, S.; Gênova, D. H. M.; Brito, I. A. O.; Lima, A. R. F.; Nobre, M. A. L.; J. Solid State Chem. 2011, 184, 990.

23. Azough, F.; Freer, R.; Thrall, M.; J. Eur. Ceram. Soc. 2010, 30, 727.

24. Chandra Sati, P.; Arora, M.; Chauhan, S.; Kumar, M.; Chhoker, S.; Ceram. Int. 2014, 40, 7805.

25. Farmer, V. C.; Infrared Spectra of Minerals, Mineralogical Society of Great Britain \& Ireland, 1977.

26. Reddy, R.; Rao, R.; Viswanath, R.; J. Am. Chem. Soc. 1989, 111, 2914.

27. Watcharapasorn, A.; Jiansirisomboon, S.; Tunkasiri, T.; Mater. Lett. 2007, 61, 2986.

28. Martin, L. W.; Chu, Y-H.; Ramesh, R.; Mater. Sci. Eng., R. 2010, 68, 89.

29. Rodrigues, H. O.; Pires, G. F. M.; Almeida, J. S.; Sancho, E. O.; Ferreira, A. C.; Silva, M. A. S.; Sombra, A. S. B.; J. Phys. Chem. Solids 2010, 71, 1329.

30. Das, R.; Mandal, K.; J. Magn. Magn. Mater. 2012, 324, 1913.

31. Cheng, G. F.; Ruan, Y. J.; Liu, W.; Wu, X. S.; Phys. B (Amsterdam, Neth.) 2015, 468-469, 81 .

32. Deng, X.; Huang, J.; Zhang, Y.; Cai, W.; Fu, C.; Ferroelectrics 2015 , $478,11$.

33. Yang, C.; Liu, C. Z.; Wang, C. M.; Zhang, W. G.; Jiang, J. S.; J. Magn. Magn. Mater. 2011, 324, 1483.

34. Singh, H.; Yadav, K. L.; Mat. Chem. Phys. 2012, 132, 17.

35. Wu, M. S.; Huang, Z. B.; Han, C. X.; Yuan, S. L.; Lu, C. L.; Xia, S. C.; Solid State Commun. 2012, 152, 2142.

36. Godara, S.; S. Kumar, S.; Ceram. Inter. 2015, 41, 6912.

37. Pal, V.; Dwivedi, R. K.; Thakur, O. P.; Curr. Appl. Phys. 2014, 14, 99.

38. Warren, B. E.; X-ray diffrraction, Addison Wesley: U.S.A., 1990.

39. Wilson, A. J. C.; X-ray Optics. The diffraction of X-rays by finite and imperfect crystals, John Wiley \& Sons Inc.: New York, 1949.

40. Aguiar, E. C.; Ramirez, M. A.; Moura, F.; Varela, J. A.; Longo, E.; Simões, A. Z.; Ceram. Int. 2013, 39, 13.

41. Srivastava, A.; Garg, A.; Morrison, F. D.; J. Appl. Phys. 2009, 105, 054103.

42. Kumar, A.; Singh, B. P.; Choudhary, R. N. P.; Thakur, A. K.; Mat. Chem. Phys. 2006, 99, 150.

43. Mishra, A.; Choudhary, S. N.; Prasad, K.; Choudhary, R. N. P.; Phys. B (Amsterdam, Neth.) 2011, 406, 3279.

44. Das, P. R.; Pati, B.; Sutar, B. C.; Choudhary, R. N. P.; Adv. Mater. 2012, 3,8 .

45. Kumar, A.; Choudhary, R. N. P.; Singh, B. P.; Takur, A. K.; Ceram. Int. 2006, 32, 73 .

46. Coondoo, I.; Panwar, N.; Tomar, A.; Jha, A. K.; Agarwal, S. K.; Phys. B (Amsterdam, Neth.) 2012, 407, 4712.

47. Nattaya, T.; Sutjarittangtham, K.; Tunkasiri, T.; Pengpat, K.; Rujijanagul, G.; Wang, J.; Ceram. Int. 2013, 39, S145.

48. Bahuguna Saradhi, B. V.; Srinivas, K.; Prasad, G.; Suryanarayana, S. V.; Bhimasankaram, T.; Mat. Sci. Eng., B. 2003, 98, 10.

49. Jia, D-C.; Xu, J-H.; Ke, H.; Wang, W.; Zhou, Y.; J. Eur Ceram Soc. 2009, 29, 3099.

50. Karthik, T.; Srinivas, A.; Kamaraj, V.; Chandrasekeran, V.; Ceram. Int. 2012, 38, 1093.

51. Luo, L.; Wei, W.; Yuan, X.; Shen, K.; Xu, M.; Xu, Q.; J. Alloys Compd. 2012, 540, 36 .

52. Wei, J.; Xue, D.; Appl. Surf. Sci. 2011, 258, 1373.

53. Bhushan, B.; Basumallick, A.; Vasanthacharya, N. Y.; Kumar, S.; Das, D.; Solid State Sci. 2010, 12, 1063. 